\title{
COVID-19 e instituciones de cuidados a largo plazo en México: una deuda impostergable
}

\author{
Jorge A. Mena-Madrazo, ${ }^{1}$ Eduardo Sosa-Tinoco, ${ }^{2 *}$ Miguel Flores-Castro, ${ }^{3}$ Mariana López-Ortega ${ }^{4}$ y \\ Luis M. Gutiérrez-Robledo 5 \\ ${ }^{1}$ Residencia Vita et Sofia, Dirección Médica, Ciudad de México; ${ }^{2}$ Secretaría de Salud, Instituto Nacional de Geriatría, Dirección de Enseñanza y \\ Divulgación, Ciudad de México; ${ }^{3}$ Antiguo Hospital Civil de Guadalajara "Fray Antonio Alcalde", Servicio de Geriatría, Jalisco; " Instituto Nacional de \\ Geriatría, Dirección de Investigación, Ciudad de México; ${ }^{5}$ nstituto Nacional de Geriatría, Dirección General, Ciudad de México. México
}

\section{Resumen}

Ante la pandemia de COVID-19, el grupo más afectado ha sido el de las personas mayores que viven en instituciones de cuidados a largo plazo (ICLP), el cual ha acumulado entre 30 y $60 \%$ de los fallecimientos en el mundo. En México se han reportado brotes en residencias de por lo menos ocho entidades federativas. Diversos factores hacen susceptibles a este grupo y a las ICLP: la convivencia en lugares comunes, dormitorios compartidos y el contacto físico con el personal que ahí labora, aunados a la falta de protocolos y estándares de cuidados de observancia obligatoria, así como a la escasa capacitación del personal. Se evidencia la necesidad de desarrollar un Sistema Nacional de Cuidados que brinde apoyo a las personas con dependencia de cuidados y sus familias y que incluya a las ICLP. Derivado de los retos por la carencia de información y competencias en prevención y control de infecciones en las ICLP, un conjunto de expertos, en colaboración con instituciones públicas, integramos un grupo con el objetivo de actualizar las guías que permitan a las ICLP hacer frente a la pandemia y que contribuyan a la generación de ese Sistema Nacional de Cuidados.

PALABRAS CLAVE: COVID-19. Instituciones de cuidados a largo plazo. México. Sistema Nacional de Cuidados.

\section{COVID-19 and long-term care facilities in Mexico: a debt that cannot be postponed}

\begin{abstract}
Since the emergence of the COVID-19 pandemic, the most affected population group has been that of older people living in long-term care facilities (LTCFs), which has accumulated between 30 and $60 \%$ of total number of deaths in the world. In Mexico, outbreaks have been reported in LTCFs of at least eight states. Various factors make this population group and LTCFs susceptible to COVID-1 outbreaks, mainly due to coexistence in common spaces, shared bedrooms and permanent physical contact with the personnel who work there, coupled with a lack of protocols and standards of care of mandatory observance, as well as personnel training limitations. There is evidence of the need to formally develop a National Care System that provides support to those in need of care and their families, and that includes LTCFs. In view of the challenges due to the lack of information and competencies in infection prevention and control at LTCFs, a group of experts, in collaboration with different public institutions, joined efforts with the purpose to update the guidelines in order to allow LTCFs face the pandemic and to contribute to the generation of said National Care System.
\end{abstract}

KEY WORDS: COVID-19. Long-term care facilities. Mexico. National Care System.

Correspondencia:

*Eduardo Sosa-Tinoco

E-mail: esosa@inger.gob.mx

0016-3813/С 2020 Academia Nacional de Medicina de México, A.C. Publicado por Permanyer. Este es un artículo open access bajo la licencia CC BY-NC-ND (http://creativecommons.org/licenses/by-nc-nd/4.0/).

Fecha de recepción: 05-08-2020

Fecha de aceptación: 17-08-2020

DOI: 10.24875/GMM.20000549
Gac Med Mex. 2021;157:99-102

Disponible en PubMed

www.gacetamedicademexico.com 
Uno de los sectores más afectados por la pandemia de COVID-19 son las personas mayores que viven en instituciones de cuidados a largo plazo (ICLP). La Organización Mundial de la Salud estima que en muchos países más de $40 \%$ de las muertes relacionadas con COVID-19 ha ocurrido en ICLP. En Estados Unidos, para mayo de 2020 el virus había infectado a más de 153000 personas y tomado la vida de más de 2800 residentes y trabajadores de estas instituciones. ${ }^{2}$ En junio de 2020 se creó un registro estadounidense de COVID-19 en ICLP, con 152281 casos confirmados y 40273 muertes para julio de $2020 .{ }^{3}$ En algunos países europeos se estima que 30 a $60 \%$ de los fallecidos durante la pandemia eran residentes de ICLP. ${ }^{4}$

Las características de estas instituciones las hacen particularmente susceptibles a brotes de COVID-19: la convivencia en lugares comunes, la cercanía física entre las personas, el importante número de ellas que requiere asistencia para realizar actividades diarias y que, por lo tanto, está en contacto físico con el personal. Frecuentemente los residentes de esos centros padecen múltiples enfermedades crónicas, factor de riesgo para mayor gravedad y mortalidad por COVID-19. A esto se suman factores organizacionales como la falta de protocolos y estándares de cuidados, de infraestructura y recursos para el aislamiento, así como la limitada capacitación del personal. $^{5}$

El censo de establecimientos de asistencia social de 2015 mostró que solo $1 \%$ del personal que labora en casas hogar para ancianos contaba con certificación de competencias sobre atención a esta población. En este censo se identificaron 1020 asilos, donde residían 22611 personas mayores, $61 \%$ mujeres y $39 \%$ hombres; $60 \%$ tenía 80 años o más. ${ }^{6}$ Existe, además, un número indeterminado de "casas hogar" no registradas.

El Estudio Nacional de Salud y Envejecimiento en México demuestra que aproximadamente $14 \%$ de las personas mayores que viven en comunidad tienen limitaciones para realizar actividades básicas de la vida diaria, es decir, están en situación de dependencia de cuidados, lo cual corresponde a casi 3 millones de personas; solo $41 \%$ de ellas recibía los cuidados que requería. $^{7}$

La problemática que han tenido otros países durante la pandemia también sucede en México. Considerando los primeros 50 mil casos confirmados, cerca de la quinta parte $(21 \%)$ correspondió a personas de 60 años y más, $40 \%$ mujeres y $60 \%$ hombres; casi dos de cada tres ameritaron hospitalización (66 \%) y de ellas, 12 \% recibió ventilación mecánica. A mediados de mayo de 2020, la letalidad estimada de COVID-19 en personas mayores de México fue de $27 \% .^{8}$ En un análisis de poco más de 20 mil casos confirmados de COVID-19 en personas mayores de México se observó que la mayor gravedad y letalidad se relacionó con la comorbilidad y algunos determinantes sociales de la salud. ${ }^{9}$ Hasta julio de 2020 se habían identificado brotes de COVID-19 en ICLP de varias entidades federativas del país. ${ }^{10-17}$ Dado que existe una cantidad desconocida de asilos clandestinos, es posible que existan otros brotes no reportados.

En abril de 2020, el Instituto Nacional de Geriatría publicó recomendaciones sobre COVID-19 en ICLP; ${ }^{18}$ junto con el Instituto Mexicano del Seguro Social, integró un curso para el público en general. ${ }^{19}$ Con más de 25000 personas inscritas en los primeros días, el Instituto Nacional de Geriatría inició el levantamiento de una encuesta ${ }^{20}$ orientada a medir la preparación y la respuesta de las ICLP ante la pandemia, para lo cual estableció contacto con cientos de esas instituciones; además, tradujo al español una estrategia sobre prevención y control de COVID-19 en ICLP. ${ }^{21}$

Debido a los grandes retos por la carencia de información y competencias en prevención y control de infecciones en las ICLP, en mayo de 2020, geriatras asociados al Colegio Nacional de Medicina Geriátrica, convocados por el Instituto Nacional de Geriatría, se reunieron para integrar un grupo de expertos con el objetivo de actualizar las guías para que las ICLP hagan frente a la pandemia. Se desarrolló un curso en línea dirigido al personal que labora en ICLP. ${ }^{22}$ Lo anterior representó un evento significativo en la geriatría de México, pues constituyó la primera acción concertada entre profesionales de los cuidados a largo plazo con el objetivo de mejorar la calidad de la atención que se presta en estas instituciones.

Durante este tiempo nos hemos percatado de la enorme heterogeneidad de las ICLP, de la falta de protocolos estandarizados, pero también de aspectos que tienen en común y de la necesidad de que el trabajo sea continuo y no solo un esfuerzo ligado a la pandemia. Durante el pico de la pandemia, el apoyo a las residencias de personas mayores se vuelve crítico pues un solo brote en una institución de mediano tamaño es capaz de saturar un hospital. Las necesidades de las ICLP continuarán después de la pandemia. 
Las ICLP deberían formar parte del sector salud porque cumplen un propósito muy específico al velar por las personas con dependencia de cuidados que por algún motivo no pueden ser cuidadas por sus familias. Actualmente, la proporción de personas mayores que viven en ICLP es pequeña, pero el acelerado envejecimiento demográfico y otros factores sociales podrían ocasionar el aumento de su número en el futuro cercano. De particular importancia es la menor disponibilidad de cuidadores familiares como resultado de familias más pequeñas y la participación de las mujeres en la fuerza laboral.

Por otra parte, la situación económica de muchas familias en el país limita la posibilidad de contar con integrantes que permanezcan en el hogar realizando actividades de cuidado, por la necesidad de incorporarse al mercado laboral. Lo anterior pone de manifiesto la necesidad de desarrollar formalmente en México un Sistema Nacional de Cuidados, en virtud de que la carga de multimorbilidad crónica y el envejecimiento de la población duplicará la prevalencia de la dependencia de cuidados en los próximos 15 años. ${ }^{23}$

La pandemia ha evidenciado las deficiencias de las ICLP a nivel global y la necesidad de una reforma y el fortalecimiento de los sistemas de cuidados con una mayor vinculación con el sector salud.

En México, no podemos eludir nuestra obligación de trabajar en el fortalecimiento de estas instituciones para que sean dignas de su misión, y, a la par, desarrollar un Sistema Nacional de Cuidados que apoye a la totalidad de las personas con dependencia de cuidados y sus familias. Es una deuda social y una necesidad creciente, reconocida en la Constitución de la Ciudad de México ${ }^{24}$ y en el Programa Sectorial de Bienestar 2020-2024..$^{25}$ En Estados Unidos se estima que se requerirán alrededor de 15,000,000,000.00 USD para que las ICLP sobrevivan a la pandemia. ${ }^{26}$ Seguramente en nuestro país no podremos movilizar semejantes recursos, pero es clara la necesidad de actuar en pro de una mejora de la calidad de la atención y un mayor reconocimiento a la labor de los trabajadores del sector.

Dentro de la tormenta surge la esperanza a partir del trabajo conjunto de los profesionales de los sectores salud y bienestar, en una colaboración intersectorial permanente dirigida a hacer de las ICLP lugares en donde se garanticen los derechos de las personas mayores y puedan vivir con la mejor calidad de vida posible.

\section{Conflicto de intereses}

Los autores declaran no tener ningún conflicto de intereses.

\section{Financiamiento}

Los autores no recibieron patrocinio para llevar a cabo este artículo.

\section{Responsabilidades éticas}

Protección de personas y animales. Los autores declaran que para esta investigación no realizaron experimentos en seres humanos ni en animales.

Confidencialidad de los datos. Los autores declaran que han seguido los protocolos de su centro de trabajo sobre la publicación de datos de pacientes.

Derecho a la privacidad y consentimiento informado. Los autores declaran que en este artículo no aparecen datos de pacientes.

\section{Bibliografía}

1. World Health Organization [Internet]. Suiza: Preventing and managing COVID-19 across long-term care services: policy brief; 2020.

2. Yourish K, Lai KKR, Ivory D, Smith M. One third of all US coronavirus deaths are in nursing home residents or workers. New York Times [Internet]. 2020 May 11.

3. Centers for Medicare \& Medicaid Services [Internet]. EE. UU.: COVID-19 nursing home data; 2020.

4. International Long Term Care Policy Network [Internet]. Comas-Herrera A Zalakaín J, Litwin C, Hsu AT, Lemmon E, Henderson D, et al. Mortality associated with COVID-19 outbreaks in care homes: early international evidence. [Actualizado 2021 Feb 01].

5. Werner RM, Hoffman AK, Coe NB. Long-term care policy after Covid-19 - solving the nursing home crisis. N Engl J Med. 2020;383;903-905.

6. Instituto Nacional de Geografía y Estadística [Internet]. México: Censo de alojamientos de asistencia social 2015. Tabulados; 2020.

7. Diagnóstico y propuesta. Cuidados a largo plazo a nivel comunitario en México. México: Instituto Nacional de Geriatría; 2019.

8. Secretaría de Salud [Internet]. México: Datos abiertos. Información referente a casos COVID-19 en México; 2020.

9. Bello-Chavolla OY, González-Díaz A, Antonio-Villa NE, Fermín-Martínez CA, Márquez-Salinas A, Vargas-Vázquez A, et al. Unequal impact of structural health determinants and comorbidity on COVID-19 severity and lethality in older Mexican adults: Considerations beyond chronological aging. J Gerontol A Biol Sci Med Sci. 2020:glaa163.

10. NL reporta nuevo brote de COVID-19 en asilo y a una bebé con el coronavirus. Animal Político [Internet]. 2020 May 17

11. Un adulto mayor fallecido y 5 graves por brotes de COVID-19 en asilos de Tabasco y NL. Animal Político [Internet]. 2020 May 7.

12. Sánchez M, Chio Y. Brote de COVID en casa hogar de Ciudad Victoria. La Jornada [Internet]. 2020 Jun 13.

13. Fallecen cuatro adultos mayores por Covid-19 en asilo de Mexicali. Aristegui Noticias [Internet]. 2020 May 12.

14. Tonantzin P. Reportan brote de Covid-19 en asilo de Morelos. Excelsior [Internet]. 2020 May 11.

15. Estrada J. Denuncian brote de Covid-19 en asilo de Chihuahua. La Jornada [Internet]. 2020 May 12.

16. Becerril J, Wong AP. Desalojan asilo en la alcaldía Cuauhtémoc por brote de coronavirus. Milenio [Internet]. 2020 May 25.

17. Brote de COVID en asilo de Coahuila deja dos mujeres muertas y 15 contagiados. Animal Político [Internet]. 2020 Jul 8. 
18. Instituto Nacional de Geriatría [Internet]. México: Prevención de enfermedad por coronavirus 2019 en residencias de personas mayores; 2020

19. Instituto Mexicano del Seguro Social [Internet]. México: CLIMSS. Nuestros cursos. Cursos para público general. COVID-19 y residencias de personas mayores; 2020. [Citado 2020 Jul 30]. Disponible en: https:// climss.imss.gob.mx

20. Instituto Nacional de Geriatría [Internet]. México: Medición y seguimiento de la preparación y respuesta local a la pandemia de COVID-19 en instituciones de cuidados a largo plazo en México; 2020

21. Instituto Nacional de Geriatría [Internet]. México: Estrategia para la prevención y el control de COVID-19 en instituciones de cuidados a largo plazo basada en delimitación explícita de zonas de riesgo; 2020.
22. Curso COVID-19 y residencias de personas mayores. México: Instituto Nacional de Geriatría; c2020.

23. Gutiérrez-Robledo LMF, García-Peña MC, Jiménez-Bolón JE, editores. Envejecimiento y dependencia. Realidades y previsión para los próximos años. México: Editorial Intersistemas/Academia Nacional de Medicina de México; 2014

24. Constitución Política de la Ciudad de México. México: Gaceta Oficial de la Ciudad de México; 2017 Feb 05.

25. Secretaría de Bienestar. Programa Sectorial de Bienestar 2020-2024. Diario Oficial de la Federación; 2020 Jun 26.

26. Goldstein M, Gebeloff R, Silver-Greenberg J. Pandemic's costs stagger the nursing home industry. New York Times [Internet]. 2020 Apr 21. 